\title{
Proposal of a Novel Index for Selection of Optimal Annuloplasty Ring Size for Tricuspid Annuloplication
}

\author{
Shuhei Fujita, MD; Hirokuni Arai, MD, PhD; Makoto Tomita, PhD; \\ Tomohiro Mizuno, MD, PhD; Satoru Kawaguchi, MD, PhD; Susumu Manabe, MD, PhD; \\ Tsuyoshi Hachimaru, MD; Naoto Miyagi, MD, PhD
}

\begin{abstract}
Background: Optimal ring size in tricuspid annuloplasty (TAP) surgery to treat functional tricuspid regurgitation (TR) was investigated because optimal ring size remains undefined.
\end{abstract}

Methods and Results: Sixty seven patients who underwent TAP at our institution were retrospectively studied. Tricuspid Annuloplasty Ring size Index (TARI) was defined as implanted tricuspid annuloplasty ring size divided by body surface area (BSA). Different TARI cut-off values were tested to determine which value produced the greatest difference in TR improvement (TRI=preoperative minus postoperative TR grade) between patients with TARI smaller (group $S$ ) and larger (group $L$ ) than the cut-off. Group $S$ was also subdivided by ring type: Cosgrove rings (SC) and MC3 rings (SM). TARI and TRI were negatively correlated $(r=-0.307)$. A TARI threshold of $18.9 \mathrm{~mm} / \mathrm{m}^{2}$ produced the greatest and most significant difference $(P<0.0005)$ in TRI. Defining groups $S$ and $L$ using this threshold, TRI was significantly greater for group $S(1.77 \pm 0.80)$ than for group $L(0.97 \pm 0.83) ; P<0.0005$. There was no difference in TRI between groups SC and SM.

Conclusions: A novel index TARI that normalizes tricuspid annuloplasty ring size by BSA was developed. Choosing ring size to make TARI $<18.9 \mathrm{~mm} / \mathrm{m}^{2}$ is likely to be better than setting an upper limit of absolute ring size in the surgical treatment of TR. (Circ $J$ 2013; 77: 2505-2513) Key Words: Annuloplasty ring; Body surface area; Look-up table of ring size; Tricuspid annuloplication; Tricuspid
regurgitation

$\mathbf{F}$ unctional tricuspid regurgitation (TR) arises when the tricuspid valve (TV) annulus dilates as a part of right ventricular enlargement secondary to left-sided heart disease. ${ }^{1}$ Untreated functional TR would result in reoperation in some cases. ${ }^{2}$ Surgical correction of functional TR at the time of mitral or aortic valve operation is well-accepted, ${ }^{3-8}$ but to date, there is no consensus regarding optimal procedure or selection of annuloplasty ring size when treating TR. ${ }^{9-16}$ Tricuspid annuloplication (TAP) with a TV annuloplasty ring is the most common operation undertaken at present, but the rate of TR control failure is quite high (15-30\%). ${ }^{10-16}$ Use of an undersized ( 26 or $28 \mathrm{~mm}$ ) tricuspid annuloplasty ring has been recommended to achieve consistent TR regulation. ${ }^{16}$ However, there are some patients in whom we implant a greater size ring, for example $30-34 \mathrm{~mm}$, to reduce the necessity for reoperation of TV. We believe that there is likely to be an optimal ring size for a given patient, but have found empirical evaluation of annular diameter to be unreliable. Criteria for selecting ring size based on some objective measure would be highly useful. The aim of our study was to test whether normalizing tricuspid annuloplasty ring size by body surface area (BSA) could help in this regard. Normalizing by BSA has been recommended as a method to eliminate variability in outcomes that are due to differences in cardiac size. ${ }^{18}$

\section{Methods}

Our study cohort consisted of 67 consecutive patients who underwent TV repair with annuloplasty rings for functional TR from January 2006 to September 2011 at our institution. We retrospectively identified patients using our local clinical database. In our database, BSA was calculated as described by $\mathrm{Du}$ Bois and DuBois IV:19

$$
\text { BSA }\left(\mathrm{m}^{2}\right)=\mathrm{BW}^{0.425}(\mathrm{~kg}) \times \mathrm{BL}^{0.725}(\mathrm{~cm}) \times 0.007184
$$

where BW is weight and BL is height. Preoperative TR grade

Received March 6, 2013; revised manuscript received May 8, 2013; accepted June 7, 2013; released online July 9, 2013 Time for primary review: 43 days

Department of Cardiovascular Surgery, Tokyo Medical and Dental University, Tokyo (S.F., H.A., T.M., S.K., S.M., T.H.); Tokyo Medical and Dental University Hospital of Medicine, Clinical Research Center, Tokyo (M.T.); and Department of Cardiovascular Surgery, Machida Municipal Hospital, Tokyo (N.M.), Japan

Mailing address: Hirokuni Arai, MD, PhD, Department of Cardiovascular Surgery, Tokyo Medical and Dental University, 1-5-45 Yushima, Bunkyo-ku, Tokyo 113-8510, Japan. E-mail: hiro.cvsg@tmd.ac.jp

ISSN-1346-9843 doi:10.1253/circj.CJ-13-0324

All rights are reserved to the Japanese Circulation Society. For permissions, please e-mail: cj@j-circ.or.jp 
Table. Patients Characteristics of Total Patients and Patients of Group S and Group $L$

\begin{tabular}{|c|c|c|c|c|}
\hline Variables $(\mathrm{N}=67)$ & Total patients & Group S ( $n=35)$ & Group L $(n=32)$ & $P$ value \\
\hline Preoperative characteristics & $\begin{array}{c}\text { Mean } \pm \text { SD (Range) } \\
\text { or No. }(\%)\end{array}$ & & & \\
\hline Age, years & $62.7 \pm 11.4$ & $62.4 \pm 11.9(28-79)$ & $62.9 \pm 11.2(35-80)$ & 0.87 \\
\hline Female sex & $36(53.7 \%)$ & $14(40 \%)$ & $22(69 \%)$ & 0.015 \\
\hline BSA & $1.57 \pm 0.17$ & $1.66 \pm 0.15$ & $1.48 \pm 0.12$ & 0.0000004 \\
\hline \multicolumn{5}{|l|}{ NYHA class } \\
\hline 1 & $10(14.9 \%)$ & $8(23 \%)$ & $2(6.3 \%)$ & 0.056 \\
\hline$\|$ & $38(56.7 \%)$ & $18(51 \%)$ & $20(63 \%)$ & 0.37 \\
\hline III & $14(20.9 \%)$ & $8(23 \%)$ & $6(19 \%)$ & 0.69 \\
\hline IV & $5(7.5 \%)$ & $1(2.9 \%)$ & $4(13 \%)$ & 0.14 \\
\hline Average NYHA function & $2.2 \pm 0.87$ & $2.1 \pm 0.76$ & $2.4 \pm 0.79$ & 0.1 \\
\hline Left ventricular ejection fraction, \% & $59.3 \pm 13.8$ & $59 \pm 14$ & $61 \pm 14$ & 0.6 \\
\hline Systolic pulmonary artery pressure $(\mathrm{mmHg})$ & $37.5 \pm 14.8$ & $34 \pm 12.2$ & $41.3 \pm 16.6$ & 0.043 \\
\hline Atrial fibrillation & $53(79.1 \%)$ & $30(86 \%)$ & $23(72 \%)$ & 0.17 \\
\hline Mitral valve disease & $60(89.6 \%)$ & $31(88.6 \%)$ & $29(90.6 \%)$ & 0.90 \\
\hline Aortic valve disease & $21(60.0 \%)$ & $12(34.3 \%)$ & $9(93.8 \%)$ & 0.066 \\
\hline \multicolumn{5}{|l|}{ Tricuspid regurgitation grade } \\
\hline Mild & $7(10.4 \%)$ & $4(11 \%)$ & $3(9.3 \%)$ & 0.79 \\
\hline Mild to moderate & $8(11.9 \%)$ & $6(17 \%)$ & $2(6.3 \%)$ & 0.17 \\
\hline Moderate & $24(35.8 \%)$ & $9(26 \%)$ & $15(47 \%)$ & 0.07 \\
\hline Moderate to severe & $10(14.9 \%)$ & $7(20 \%)$ & $3(9.3 \%)$ & 0.23 \\
\hline Severe & $18(26.9 \%)$ & $9(26 \%)$ & $9(28 \%)$ & 0.83 \\
\hline Average TR grade & $3.18 \pm 0.64$ & $3.16 \pm 0.67$ & $3.20 \pm 0.62$ & 0.77 \\
\hline \multicolumn{5}{|l|}{ Operative characteristics } \\
\hline Perfusion time, $\min$ & $256.4 \pm 84.2$ & $268 \pm 90$ & $244 \pm 77$ & 0.24 \\
\hline Cross-clamp time, $\min$ & $123.7 \pm 85.6$ & $132 \pm 82$ & $115 \pm 90$ & 0.43 \\
\hline Concomitant procedure & $67(100 \%)$ & $35(100 \%)$ & $32(100 \%)$ & - \\
\hline MV operation & $58(86.6 \%)$ & $29(82.9 \%)$ & $29(90.6 \%)$ & 0.34 \\
\hline $\mathrm{AV}$ replacement & $15(22.4 \%)$ & $9(25.7 \%)$ & $6(18.8 \%)$ & 0.5 \\
\hline 3 valves $(M V+T V+A V)$ & $15(22.4 \%)$ & $9(25.7 \%)$ & $6(18.8 \%)$ & 0.5 \\
\hline Atrial septal defect closure & $10(14.9 \%)$ & $5(14.3 \%)$ & $5(15.6 \%)$ & 0.88 \\
\hline Other & $4(6.0 \%)$ & $1(2.9 \%)$ & $3(9.4 \%)$ & 0.27 \\
\hline \multicolumn{5}{|l|}{ Implanted tricuspid ring size $(\mathrm{mm})$} \\
\hline 26 & $13(19.4 \%)$ & $12(34.3 \%)$ & $2(6.3 \%)$ & 0.0043 \\
\hline 28 & $14(20.9 \%)$ & $12(34.3 \%)$ & $2(6.3 \%)$ & 0.0043 \\
\hline 30 & $17(25.4 \%)$ & $3(8.6 \%)$ & $14(43.8 \%)$ & 0.0007 \\
\hline 32 & $18(26.9 \%)$ & $6(17.1 \%)$ & $11(34.4 \%)$ & 0.11 \\
\hline 34 & $5(7.5 \%)$ & $2(5.7 \%)$ & $3(9.4 \%)$ & 0.58 \\
\hline Average ring size & $29.6 \pm 2.5$ & $28.7 \pm 2.6$ & $30.7 \pm 1.9$ & 0.00066 \\
\hline Average TARI, ring size / each patient's BSA & $19.0 \pm 2.2$ & $17.3 \pm 1.4$ & $20.8 \pm 1.3$ & $<0.00001$ \\
\hline Rigid ring, $\mathrm{MC} 3$ & $33(49.3 \%)$ & $19(54.3 \%)$ & $14(43.8 \%)$ & 0.39 \\
\hline \multicolumn{5}{|l|}{ Changes in TR and MR grade } \\
\hline Average observational period, days & $371 \pm 422(7-1531)$ & $317 \pm 338(9-1207)$ & $407 \pm 499(7-1531)$ & 0.39 \\
\hline Preoperative MR grade & $3.07 \pm 1.05$ & $3.13 \pm 0.84$ & $3.02 \pm 1.25$ & 0.8 \\
\hline Postoperative MR grade & $1.00 \pm 0.86$ & $1.01 \pm 0.96$ & $0.98 \pm 0.75$ & 0.19 \\
\hline Follow up MR grade & $1.21 \pm 0.89$ & $1.04 \pm 0.88$ & $1.34 \pm 0.89$ & 0.19 \\
\hline Preoperative TR grade & $3.18 \pm 0.64$ & $3.16 \pm 0.67$ & $3.20 \pm 0.62$ & 0.8 \\
\hline Postoperative TR grade & $1.78 \pm 0.91$ & $1.40 \pm 0.82$ & $2.20 \pm 0.81$ & 0.00024 \\
\hline Follow up TR grade & $1.79 \pm 0.82$ & $1.39 \pm 0.67$ & $2.23 \pm 0.74$ & 0.000016 \\
\hline Final TR improvement & $1.41 \pm 0.93$ & $1.77 \pm 0.80$ & $0.97 \pm 0.83$ & 0.00025 \\
\hline
\end{tabular}

AV, aortic valve; BSA, body surface area; MR, mitral regurgitation; MV, mitral valve; NYHA, New York Heart Association; TARI, tricupid annuloplasty ring size index; TR, tricuspid regurgitation; TV, tricuspid valve. 
A

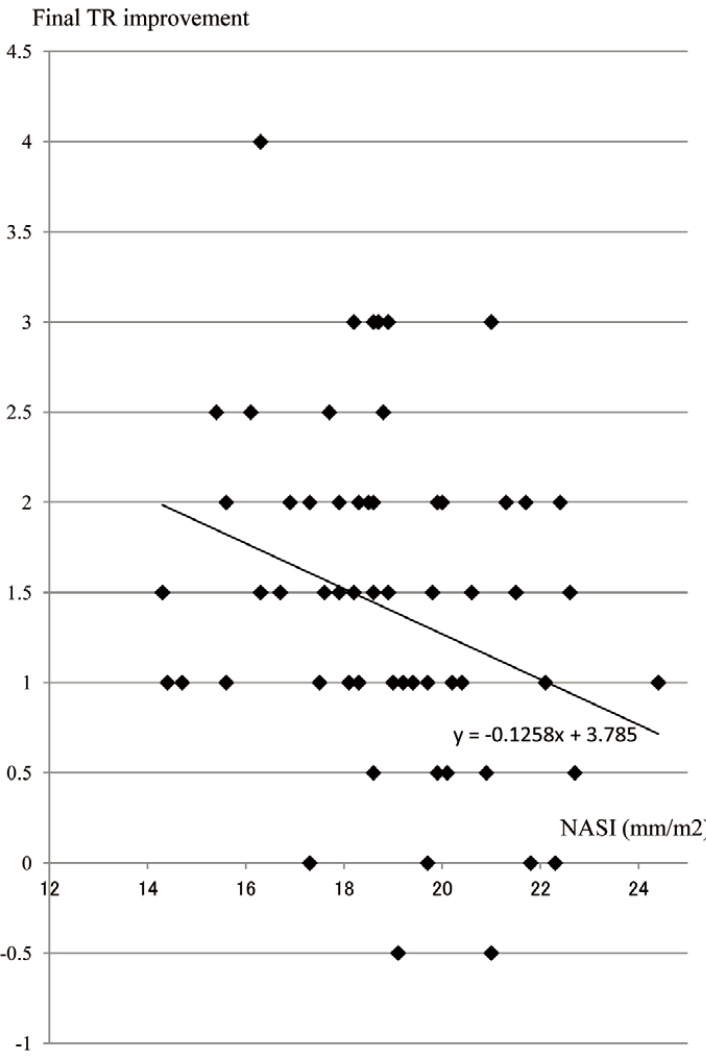

B

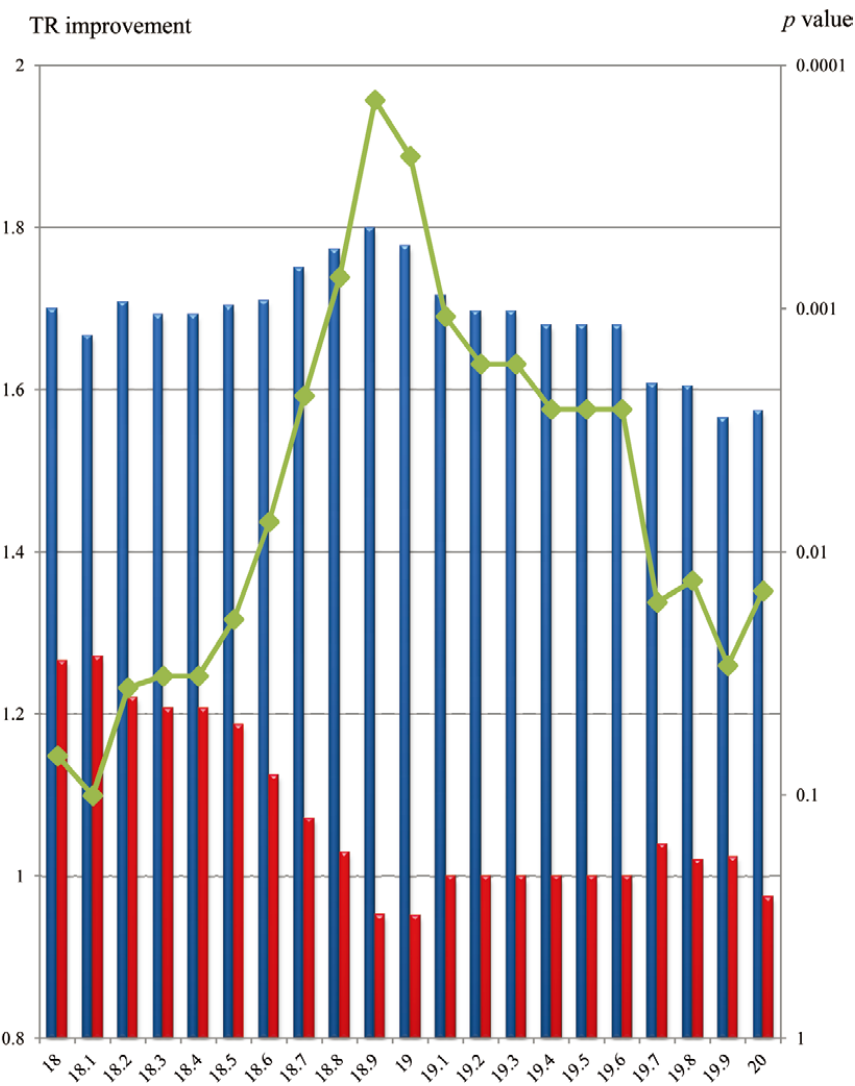

Figure 1. (A) Relationship between final tricuspid regurgitation (TR) improvement and TARI. Tricuspid Annuloplasty Ring size Index (TARI) was obtained by dividing the tricuspid annuloplasty ring size implanted in a patient by the patient's body surface area (BSA). A significantly negative correlation between TARI and TR Improvement (preoperative TR grade minus most recent TR grade) was found (coefficient of correlation=-0.307). Smaller TARI patients were likely to have greater TR improvement. (B) The comparison of TR Improvement and P values for each threshold. This figure was derived from (A). For a given threshold in (A), patients were divided into 2 groups; those with TARI smaller or larger than this threshold. The difference in TR Improvement and the P value of the difference between these 2 groups was calculated for 21 such thresholds. The blue bars represent TR Improvement for patients with TARI smaller than the threshold, and the red bars represent patients with TARI larger than the threshold. The line shows the P values for the comparison. TARI $=18.9 \mathrm{~mm} / \mathrm{m}^{2}$ gave the smallest $P$ value of 0.000139 . TARI, Tricuspid Annuloplasty Ring size Index.

was assessed in all patients with $2 \mathrm{D}$ transthoracic echocardiography (TTE) using standard methods established by the American Society of Echocardiography.$^{20}$ Preoperative TTE was performed less than 3 weeks before the operation. TR was graded on a numerical scale from 0 to $4(0=$ none, $1=$ trivial, $2=$ mild, $2.5=$ mild to moderate, $3=$ moderate, $3.5=$ moderate to severe, $4=$ severe). Postoperative TR grade was evaluated in all patients within 2 weeks of the operation. Transesophageal echocardiography (TEE) was performed preoperatively at the time of operation. Indications for TV repair included the presence of mild to moderate or greater TR grade on any preoperative TV assessment. Systolic pulmonary artery pressure (sPAP) was measured using a Swan-Ganz catheter during the operation.

In all patients, either of the 2 types of TV annuloplasty rings, a Cosgrove-Edwards Annuloplasty System or an Edwards MC $^{3}$ Tricuspid Annuloplasty Ring (Edwards Lifesciences, LLC, Irvine, CA, USA), was used to correct TR. A Cosgrove ring is a flexible annuloplasty band indicated for both mitral valve and $\mathrm{TV}$ repair, whereas an $\mathrm{MC}^{3}$ ring is a rigid $3 \mathrm{D}$ annuloplasty ring indicated for TR only. Size and type of ring to be implanted was determined by the surgeon during the operation according to visual and manual evaluation of physical features of the TV in each patient, such as presence of calcification and stiffness.

To evaluate the effect of ring size on TR control, we defined an index that we called TARI, which stands for Tricuspid Annuloplasty Ring size Index. It was obtained by dividing the size of the tricuspid annuloplasty ring implanted in each patient by his or her BSA.

TARI $\left(\mathrm{mm} / \mathrm{m}^{2}\right)=$ Implanted annuloplasty ring size $(\mathrm{mm}) /$ $\operatorname{BSA}\left(\mathrm{m}^{2}\right)$

Improvement in TR (TRI) was assessed as the difference between pre- and post-operative TR grade.

TRI= preoperative TR grade - the most recently available TR grade

Evaluation of cut-off values described in more detail in the Results section showed that a TARI of $18.9 \mathrm{~mm} / \mathrm{m}^{2}$ produced the most significant difference in TRI between groups. We then used this threshold to divide patients into 2 groups, $\mathrm{S}$ for small 


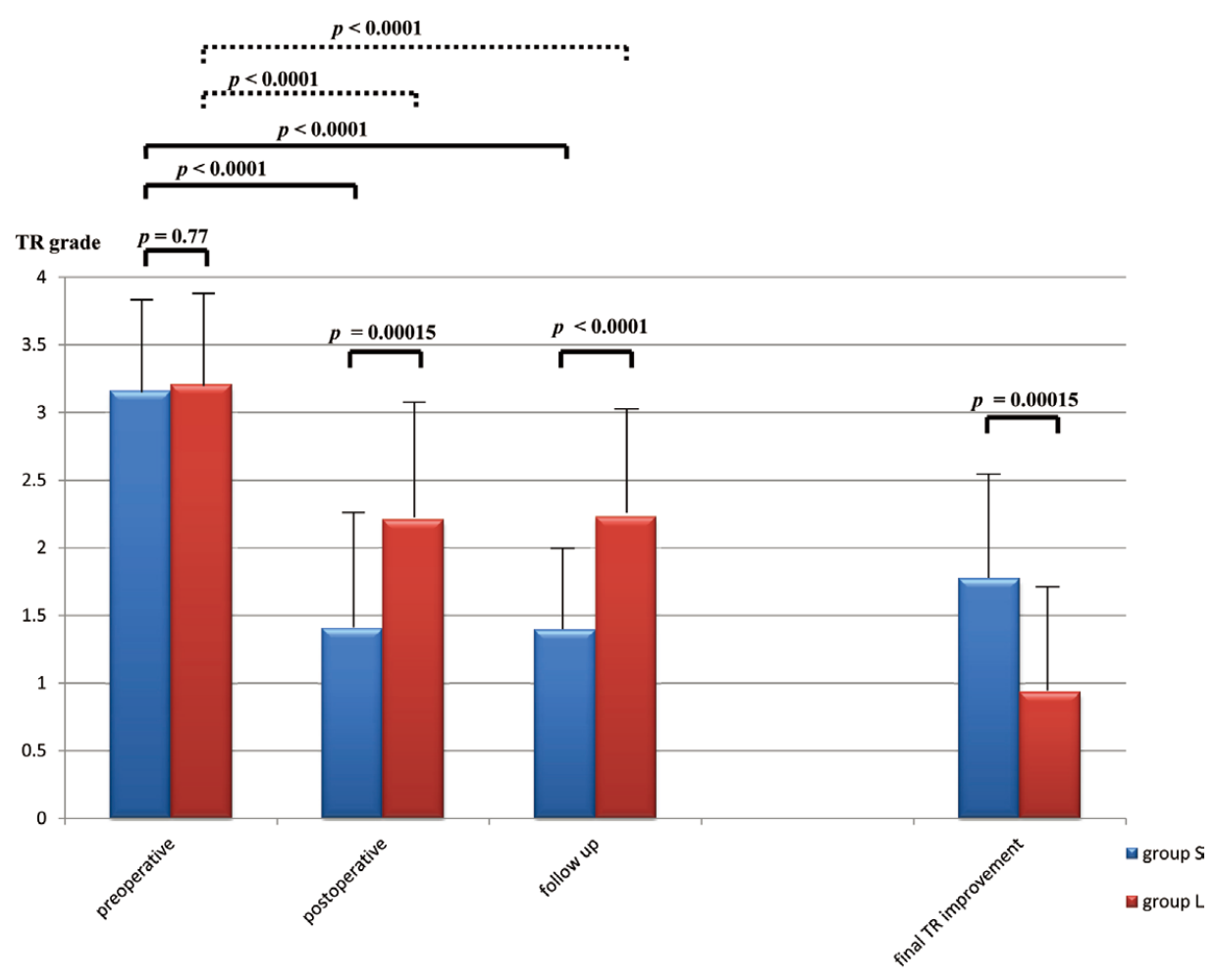

Figure 2. Comparison of tricuspid regurgitation (TR) grade and TR Improvement in group S and group L. Mean and SD of preoperative, post-operative (less than 2 weeks after surgery) and follow-up tricuspid regurgitation grades are shown for group $S$ (TARI $<1$ $8.9 \mathrm{~mm} / \mathrm{m}^{2}$ ) and group L (TARI $>18.9 \mathrm{~mm} / \mathrm{m}^{2}$ ) patients, as well as TR Improvement. Both groups showed significant improvement of TR compared with preoperative TR grade. Nevertheless, TR Improvement in group S was significantly greater than in group L.

TARI (TARI $\leq 18.9, \mathrm{n}=35)$ and $\mathrm{L}$ for large TARI (TARI $>18.9$, $\mathrm{n}=32$ ). In addition, to assess the influence of ring type on outcome, group S was further divided into groups SC (Cosgrove ring, $\mathrm{n}=16)$ and $\mathrm{SM}\left(\mathrm{MC}^{3}\right.$ ring, $\left.\mathrm{n}=19\right)$ according to which type of annuloplasty ring had been used. We evaluated TR grade from the last follow-up echocardiogram available, that is, for the few patients lost to follow up, postoperative TR grade evaluated within 2 weeks of the operation was used, while for the remaining patients, TR grade from their last follow-up exam was used.

\section{Operative Technique}

All TV repairs were performed under cardiac arrest. Intermittent anterograde and retrograde cardioplegia were used for cardiac protection. The TV was exposed through an oblique right atriotomy. The TV structure was carefully assessed to confirm the presence of functional TR. ${ }^{21}$ For all patients, a Cosgrove ring or $\mathrm{MC}^{3}$ ring was used to repair the TV. When using a Cosgrove ring, 1 suture was placed on the septal segment to make its effective orifice identical to $\mathrm{MC}^{3}$ 's one. If additional procedures (eg, commissural closure, edge-to-edge technique) were performed to repair the TV, those patients were excluded from this study to control for the difference in outcomes that might be due to auxiliary repairs. All patients underwent intraoperative TEE assessment before and after weaning from cardiopulmonary bypass.

\section{Statistical Analysis}

All analyses were performed using SPSS software (version
10.1; SPSS, Chicago, IL, USA). Values are presented as mean \pm standard deviation (SD). For bivariate comparison of the 2 groups, we used the $\chi^{2}$ test for categorical data and a nonparametric Mann-Whitney's U test for metric data. We performed Kaplan-Meier analysis of the freedom from moderate to severe or severe TR grade, followed by a log-rank test. Logistic regression was used to evaluate mitral regurgitation (MR) impact on recurrent TR. A value of $\mathrm{P}<0.05$ was considered significant.

\section{Results}

\section{Baseline Patient Profiles}

The preoperative and operative characteristics of the 67 patients are reported in Table. The mean age of patients was $63 \pm 11$ years, and 36 of the patients were women $(53.7 \%)$. The mean BSA was $1.57 \pm 0.17 \mathrm{~m}^{2}$. Atrial fibrillation was present in $79.1 \%$. The mean NYHA class was $2.2 \pm 0.87$. The mean TR grade was $3.2 \pm 0.6$. The mean LVEF was $59.3 \pm 13.8 \%$ and the mean sPAP was $37.5 \pm 14.8 \mathrm{mmHg}$. The CryoMaze procedure was performed in $62.7 \%$. The mean implanted tricuspid ring size was $29.6 \pm 2.5 \mathrm{~mm}$, the range was $26-34 \mathrm{~mm}$, and the mode was $32 \mathrm{~mm}$.

\section{Statistical Analysis of the Relationship Between TRI and TARI}

The TR and MR grades before and after operation are also reported in Table. The mean observation period was $371 \pm 422$ days (7-1,531 days). Postoperative TTE was performed with- 

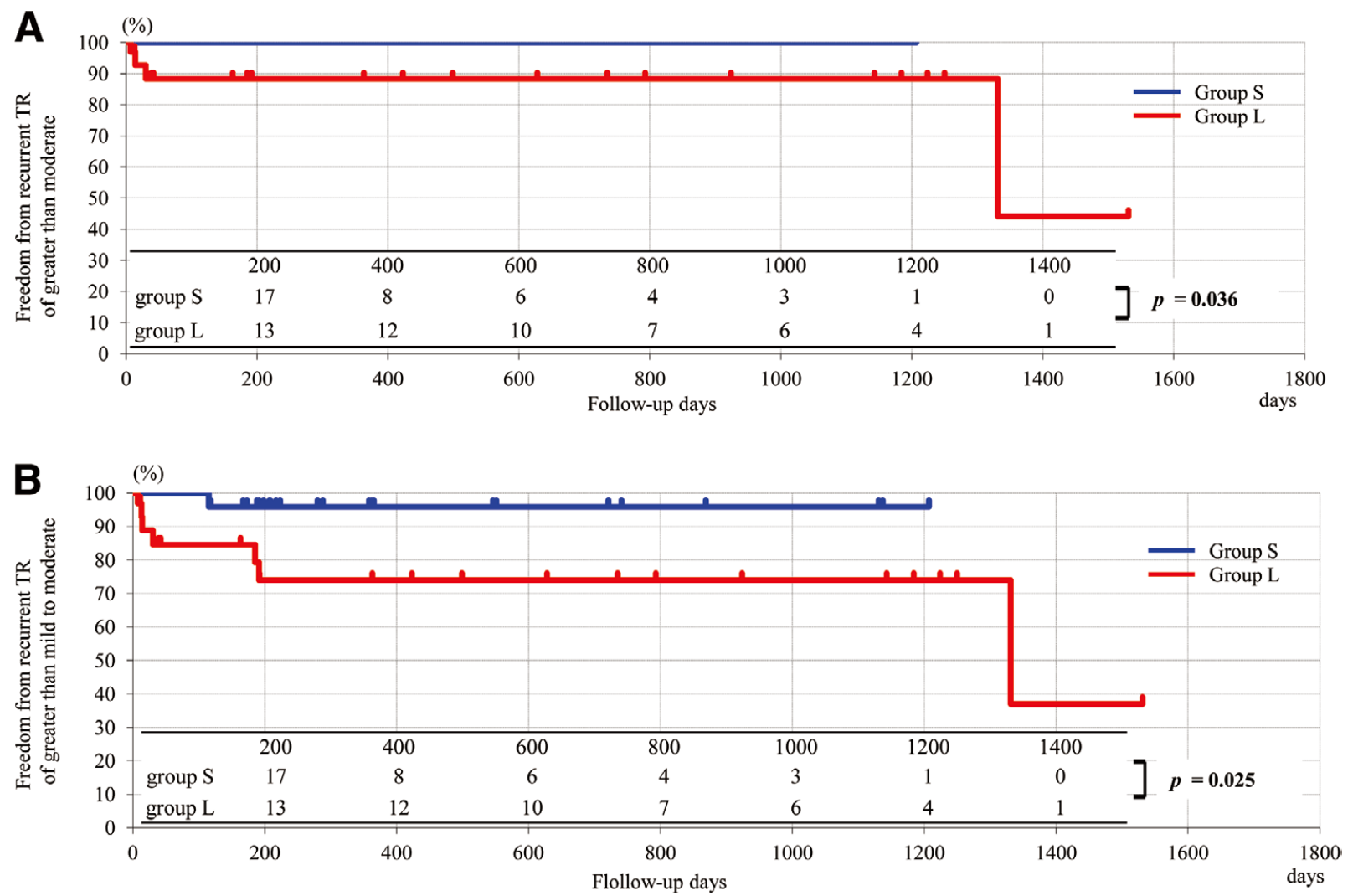

Figure 3. (A) Kaplan-Meier analysis shows freedom from tricuspid regurgitation (TR) of greater than moderate in group $S$ and group L. (B) Kaplan-Meier analysis shows freedom from TR of greater than mild to moderate in group $S$ and group $L$.

in 2 weeks of surgery in all patients and the follow-up TTE beyond 2 weeks was available for 54 out of 67 (81\%) patients. Regarding MR control, an operation improved MR grade from $3.07 \pm 1.05$ to a final grade of $1.21 \pm 0.89(\mathrm{P}<0.0001)$. All patients ended up with an MR of moderate or better grade. Regarding TR control, an operation improved TR grade from $3.18 \pm 0.64$ to a final grade of $1.79 \pm 0.82(\mathrm{P}<0.0001)$. TRI was $1.41 \pm 0.93$. Only 4 patients $(6 \%)$ had a postoperative TR of greater than moderate severity. Three of these patients were graded 3.5, and the remaining patient was graded 4. However, none of the 4 patients have required repeat surgery at the time during the writing of this paper. The postoperative TR grade from this study was relatively greater than that of other studies. This result seemed to be derived from the tendency of using larger rings for small BSA patients from 2006 to 2009.

We calculated the correlation between TRI and patient TARI (Figure 1A) and found a negative coefficient of correlation of -0.307 , that is, a smaller normalized ring size was associated with greater TRI. We then conducted the analysis as shown in Figure 1B. Briefly, after choosing a TARI threshold in Figure 1A, patients were divided into 2 groups, those whose TARI was smaller or larger than the chosen threshold. Statistical difference in TRI between these 2 groups was calculated. Figure 1B was constructed by repeating this process for 21 thresholds from $18.0 \mathrm{~mm} / \mathrm{m}^{2}$ to $20.0 \mathrm{~mm} / \mathrm{m}^{2}$ in steps of 0.1 . As shown in the figure, the TARI threshold for dividing patients into 2 groups whose TRI had the greatest difference was $18.9 \mathrm{~mm} / \mathrm{m}^{2}$. Moreover, this threshold produced the smallest $\mathrm{P}$ value of 0.000139 . In this analysis, the significance was defined at $\mathrm{P}<0.00238$ [0.05/21], based on Bonferroni's correction.

To evaluate the influence of residual MR on recurrent TR, we performed univariate logistic regression analysis for residual MR grade. When recurrent TR and residual MR were defined as a follow up TR grade $\geq 3$ and a follow up MR grade $\geq 3$, respectively, only 1 of 7 patients had both residual MR and recurrent TR. The odds ratio and $\mathrm{P}$ value were 2.67 and 0.97 , respectively, showing that TR recurrence was independent of residual MR.

\section{Reliability of $18.9 \mathrm{~mm} / \mathrm{m}^{2}$ as the TARI Threshold}

Taking the TARI threshold found to produce the greatest difference in TRI, so we divided patients into 2 groups, group $S$ whose TARI was smaller than $18.9 \mathrm{~mm} / \mathrm{m}^{2}$ and group L whose TARI was larger, to look for differences in baseline patient characteristics between them (Table). Demographic characteristics were similar, except that group $\mathrm{S}$ had a significantly smaller proportion of women and a smaller BSA than group S. The difference in preoperative TR grade was not significant between the groups. The percentages of coexisting mitral valve disease and aortic disease were not significantly different either. The Mean sPAP was $41.3 \pm 16.6 \mathrm{mmHg}$ in group $\mathrm{L}$, which was significantly higher than that in group $\mathrm{S}(\mathrm{P}<0.05)$.

Surgical characteristics including the size of the implanted annuloplasty ring are also shown in Table. Group $S$ patients received, on average, smaller sized TV rings $(28.7 \pm 2.6 \mathrm{~mm}$ in group $\mathrm{S}$ vs. $30.7 \pm 1.9 \mathrm{~mm}$ in group $\mathrm{L}, \mathrm{P}=0.0006)$. According to 


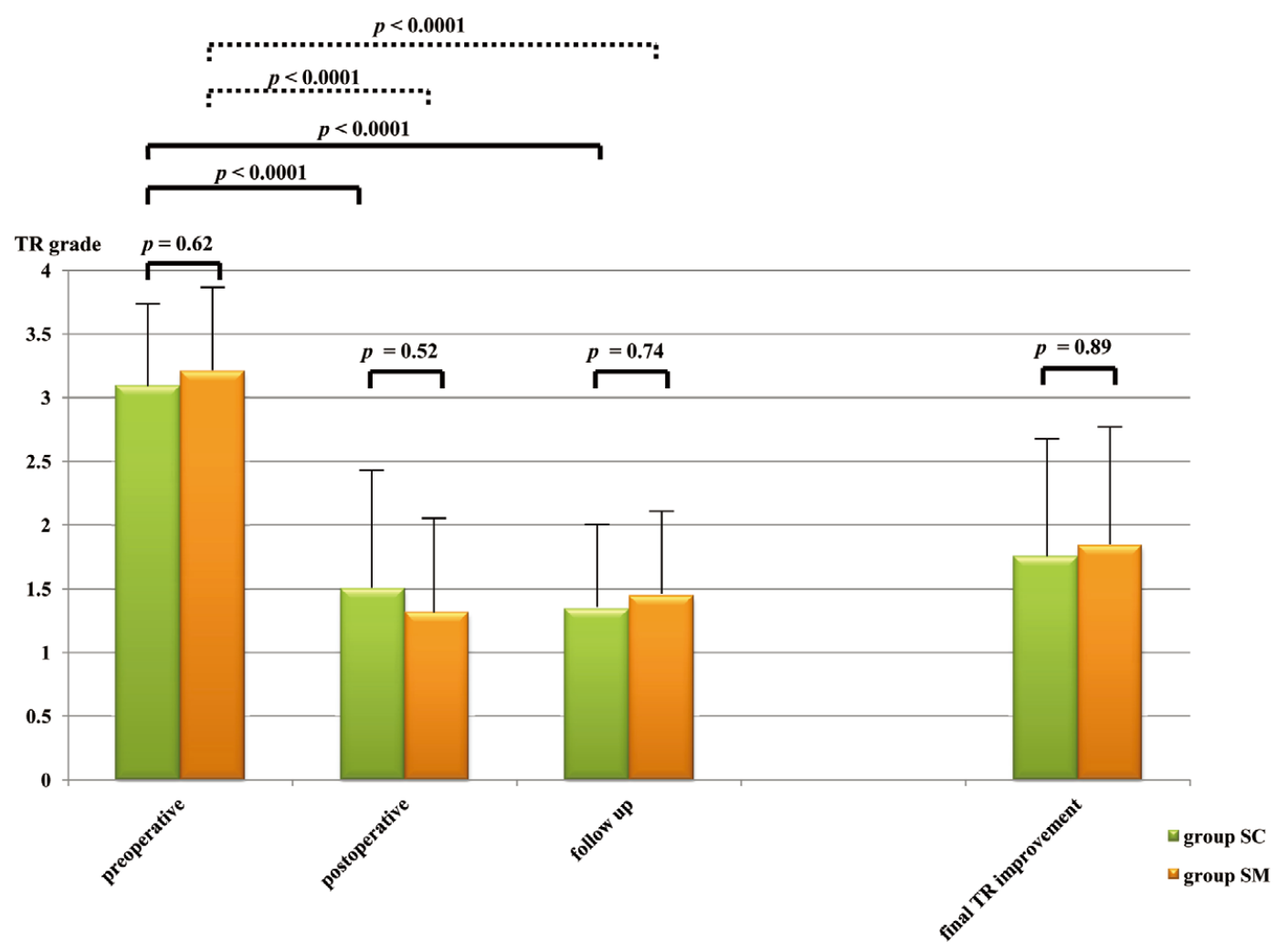

Figure 4. Tricuspid regurgitation (TR) grade and TR Improvement in group SC and group SM. Subgroup analysis of the 35 patients with TARI $<18.9 \mathrm{~mm} / \mathrm{m}^{2}$ was conducted to assess the possible influence of ring type. SC represents a Cosgrove ring, SM, represents a MC3 ring. Although TR regulation improved significantly in both subgroups after surgery, there was no significant difference between the subgroups in postoperative and follow-up TR grades, nor in TR Improvement.

crosstabs analysis, implanted ring size distribution differed significantly $(\mathrm{P}<0.0001)$ between groups $\mathrm{S}$ and L. Group $\mathrm{S}$ patients received a greater proportion of small rings.

Pre- and post- operative TR grades for groups $\mathrm{S}$ and $\mathrm{L}$ are shown graphically in Figure 2. The figure shows the similarity in preoperative TR grades $(\mathrm{P}=0.77)$ and the differences between the groups after surgery. Postoperative TR grades had improved significantly $(\mathrm{P}<0.0001$ for both groups vs. preoperative grade $)$ as had TR grade at the final follow up $(\mathrm{P}<0.0001$ for both groups vs. preoperative grade). Nevertheless, the magnitude of TRI was significantly greater for group $\mathrm{S}(\mathrm{P}<0.0005)$.

Figure 3A shows the Kaplan-Meier analysis of freedom from recurrent TR of greater than moderate severity over time. None of the 35 patients in group $S$ had a recurrent TR of greater than moderate severity ( $T R \geq 3.5$ ) compared to 4 patients out of 32 in group $L(P<0.05)$. Figure $3 B$ shows the same analysis for $\mathrm{TR} \geq 3$ and a similar result $(\mathrm{P}<0.05)$. TR regulation remained significantly better in group $\mathrm{S}$ than group $\mathrm{L}$ over the short-term follow up.

\section{Influence of Ring Properties on TRI}

We hypothesized that ring type was an additional independent factor with possible influence on TRI. However, we found no statistical difference between TR operation outcomes by ring type, whether analysis was conducted on all patients, or whether it was conducted on the subgroups S and L. Because group $\mathrm{S}$ showed the biggest TRI with surgery, we limit our descrip- tion to group $\mathrm{S}$. We divided group $\mathrm{S}(\mathrm{n}=35)$ into 2 groups; group SC consisted of the 16 patients who had received a Cosgrove ring, and group SM consisted of the 19 patients who had received an $\mathrm{MC}^{3}$ ring. In preoperative characteristics, demographics were similar, except that the mean sPAP was greater in the group SM $(\mathrm{P}<0.05)$. In terms of postoperative characteristics, the 2 groups differed significantly for mean ring size; $30.6 \pm 2.2$ vs. $27.1 \pm 1.5 \mathrm{~mm}(\mathrm{P}<0.0001)$, but this difference was made less significant when ring size was normalized by BSA; TARI was $17.9 \pm 0.85$ vs. $16.8 \pm 1.6 \mathrm{~mm} / \mathrm{m}^{2}$ $(\mathrm{P}=0.015)$. This difference seemed to be derived from the surgeon's intention to make the tricuspid annuls smaller than before from year by year.

The TR grade changes of the 2 groups are shown in Figure 4. Although surgery improved TR in both groups, as shown in Figure 2, post-operative TR grade was similar for both ring types, and the magnitude of improvement was not significantly different between the 2 groups either, signifying that ring type had little influence on TRI.

\section{Table of Recommended Tricuspid Valve Annuloplasty Ring Size for Each BSA Based on TARI}

Considering $18.9 \mathrm{~mm} / \mathrm{m}^{2}$ to be the upper limit of TARI for TR improvement, we constructed a table showing recommended TV annuloplasty ring size based on TARI (Figure 5). In this table, values of TARI are filled in for each BSA and ring size. TARI given in green and blue background cells indicate ring 


\begin{tabular}{|c|c|c|c|c|c|c|c|}
\hline \multirow[b]{2}{*}{ BSA $S I Z E$} & \multicolumn{7}{|c|}{ TARI values by annuloplasty ring size } \\
\hline & $24 \mathrm{~mm}$ & $26 \mathrm{~mm}$ & $28 \mathrm{~mm}$ & $30 \mathrm{~mm}$ & $32 \mathrm{~mm}$ & $34 \mathrm{~mm}$ & $36 \mathrm{~mm}$ \\
\hline $1.00 \mathrm{~m}^{2}$ & 24.0 & 26.0 & 28.0 & 30.0 & 32.0 & 34.0 & 36.0 \\
\hline $1.10 \mathrm{~m}^{2}$ & 21.8 & 23.6 & 25.5 & 27.3 & 29.1 & 30.9 & 32.7 \\
\hline $1.20 \mathrm{~m}^{2}$ & 20.0 & 21.7 & 23.3 & 25.0 & 26.6 & 28.3 & 30.0 \\
\hline $1.30 \mathrm{~m}^{2}$ & 18.5 & 20.0 & 21.5 & 23.1 & 24.6 & 26.2 & 27.7 \\
\hline $1.40 \mathrm{~m}^{2}$ & 17.1 & 18.6 & 20.0 & 21.4 & 22.9 & 24.3 & 25.7 \\
\hline $1.50 \mathrm{~m}^{2}$ & 16.0 & 17.3 & 18.7 & 20.0 & 21.3 & 22.7 & 24.0 \\
\hline $1.60 \mathrm{~m}^{2}$ & 15.0 & 16.3 & 17.5 & 18.8 & 20.0 & 21.3 & 22.5 \\
\hline $1.70 \mathrm{~m}^{2}$ & 14.1 & 15.3 & 16.5 & 17.6 & 18.8 & 20.0 & 21.2 \\
\hline $1.80 \mathrm{~m}^{2}$ & 13.3 & 14.4 & 15.6 & 16.7 & 17.8 & 18.9 & 20.0 \\
\hline $1.90 \mathrm{~m}^{2}$ & 12.6 & 13.7 & 14.7 & 15.8 & 16.8 & 17.9 & 18.9 \\
\hline $2.00 \mathrm{~m}^{2}$ & 12.0 & 13.0 & 14.0 & 15.0 & 16.0 & 17.0 & 18.0 \\
\hline $2.10 \mathrm{~m}^{2}$ & 11.4 & 12.4 & 13.3 & 14.3 & 15.2 & 16.2 & 17.1 \\
\hline $2.20 \mathrm{~m}^{2}$ & 10.9 & 11.8 & 12.7 & 13.6 & 14.5 & 15.5 & 16.4 \\
\hline$T A R I=R$ & Ring size / & BSA & & & ARI cutoff & $=18.9 \mathrm{~mm}$ & $n / m^{2}$ \\
\hline$\square$ TAR & l > $18.9 n$ & $\mathrm{~m} / \mathrm{m}^{2}$ & & $\square$ TARI & l $<18.9 \mathrm{~mm}$ & $/ \mathrm{m}^{2}$ & \\
\hline
\end{tabular}

Figure 5. Look-up table of recommended tricuspid valve annuloplasty ring size for each BSA based on Tricuspid Annuloplasty Ring size Index (TARI). The values in the table are TARI for each body surface area (BSA) and ring size. The cells with green and blue background indicate TARI lower than $18.9 \mathrm{~mm} / \mathrm{m}^{2}$, and ensure significant TR regulation. However, we consider the largest TARI with a blue background for a given BSA to be the most desirable to prevent stenotic conditions.

sizes that ensure significant TR regulation for each BSA. In other words, $18.9 \mathrm{~mm} / \mathrm{m}^{2}$ is the suggested maximum ring size for each BSA. This type of table should be useful and convenient in a clinical setting and might help surgeons achieve consistent TR regulation.

\section{Discussion}

The question of how to determine ring size for TAP procedures remains unsolved. Past studies have shown that smaller rings lead to better TR control, but one would like to implant the largest ring possible within such constraints to provide sufficient blood flow and prevent TV stenosis. In the present study, we defined a novel index for tricuspid annuloplasty ring size called TARI, which we calculated by dividing ring size by BSA. We found $18.9 \mathrm{~mm} / \mathrm{m}^{2}$ to be the optimal cut-off for ensuring TR improvement in TAP. Unlike proposing an upper limit of absolute ring size, using a normalized ring size takes into account differences in heart size, and allows placement of larger rings for larger hearts, and reduces the possibility of placement of too-large-a ring in the relative sense for smaller hearts. In other words, our novel index allows implantation of the largest ring possible for a given heart size.

In contrast to mitral valve repair, the reported rate of TV repair failure is relatively high. ${ }^{14-16}$ McCarthy et al reported that $15 \%$ of 790 patients who underwent TV repair for functional TR had residual TR (moderate to severe, or severe) 1 month after the operation. ${ }^{14}$ Tang et al studied 702 patients who had TV repair and found that $30 \%$ of patients had TR recurrence at a mean follow up of 5.9 years. ${ }^{11}$ Navia et al described 2,277 patients who had TV repair for functional TR. ${ }^{16}$ Eleven per cent of their patients had recurrent TR (moderate to severe, or severe) after 3 months, which increased to $17 \%$ after 5 years. Nearly $3 \%$ of the patients required a TV reoperation after 1 year. ${ }^{16}$ Fukuda et al described results of TV repair with rigid 3D annuloplasty rings in 136 patients with functional 
TR. ${ }^{22}$ Sixteen per cent of patients had moderate to severe, or severe TR in early postoperative echocardiography, and 14\% of patients eventually had TR recurrence that was greater than moderate grade. Navia et al and Fukuda et al claimed that TV repair with an annuloplasty ring could not achieve consistent TR improvement, and that therefore additional approaches addressing the sub-valvular apparatus and ventricular geometry might be necessary. ${ }^{16,22}$

In contrast, Bolling et al reported that mitral valve repair, by downsizing the mitral annulus using a flexible ring, resulted in 1 - and 2-year survival rates of $82 \%$ and $71 \%$, respectively, in 48 patients. ${ }^{23}$ Bax et al reported that $84 \%$ of 51 patients were free from residual MR 3 months after restrictive mitral annuloplasty for ischemic mitral regurgitation with downsizing of the mitral annulus. ${ }^{24}$ Kainuma et al reported that restrictive mitral annuloplication improved the NYHA class and left ventricular systolic function in $93 \%$ of 44 patients with refractory heart failure and with functional MR. ${ }^{25}$ Miura et al reported that undersized mitral annuloplasty for isolated functional MR in non-ischemic DCM improved the MR grade from 4.0 \pm 0.0 to $1.0 \pm 0.6$ in 6 patients. ${ }^{26}$ The concept of downsizing could be applied to an ascending aorta aneurysm. Hwang et al reported that 88 patients underwent reduction ascending aortoplasty and had no recurrent aortic aneurysm at a 5 year follow up. ${ }^{27} \mathrm{Ap}-$ plying this downsizing approach to TV repair, Mehrdad et al found that tricuspid annuloplasty with the use of undersized rigid 3D annuloplasty rings resulted in a very low rate of residual TR (moderate to severe, or severe). They reported that only $3 \%$ of patients had recurrent TR greater than moderate when size 26 or $28 \mathrm{~mm}$ rigid tricuspid annuloplasty rings were implanted in $88 \%$ of 101 patients. ${ }^{17}$ Filsoufi et al also supported the concept of using smaller, rigid annuloplasty rings to treat functional TR. In their study, two-thirds of 75 patients were treated with 26 or $28 \mathrm{~mm}$ rigid 3D tricuspid annuloplasty rings, and there was no significant residual TR in either early or limited midterm follow up. ${ }^{28}$ Navia et al reported that the use of a larger annuloplasty ring increased the risk of early and late recurrent TR. ${ }^{16}$ However, the appropriate threshold of ring size to treat functional TR has remained undefined.

In our study, we defined TARI to make it possible to analyze TR control under normalized conditions and were able to identify a threshold grounded in clinical data to ensure significant TR control. The choice of TV ring size or ring type was based on empirical indications, and therefore, our data set had the character of a treatment-intention study. The distribution of patients and ring size and type implanted was sufficiently spread out to tolerate statistical analysis. Our study showed that the threshold of TARI that provided the best separation of outcomes was $18.9 \mathrm{~mm} / \mathrm{m}^{2}$. In addition, this threshold had the most powerful $\mathrm{P}$ value.

Although patient numbers were relatively small, we found that there was no influence of ring type on TRI. Therefore, we suggest that the most critical factor for successful TV repair is ring size rather than ring property. Consistent with our results, Min et al studied geometric changes in the TV apparatus before and after TV annuloplasty in 59 patients using real-time 3D echocardiography. They found that preoperative tenting volume and greater anteroposterior annulus diameter $(34-40 \mathrm{~mm})$ were independent predictors of residual TR after repair. ${ }^{29} \mathrm{~A}$ plausible reason for better outcomes with undersizing the annuloplasty in functional TR is that it corrects the anteroposterior displacement of the TV annulus, improves the apposition of the TV leaflets, and relieves or reduces leaflet tethering. ${ }^{17}$ We believe that there is an optimal TV annulus size for each person and that the essential goal of TAP is to reduce the an- nulus size to that size no matter how large the patient's annulus has become.

The ring size, suggested by the threshold of TARI, we found in this study might be related to this inherent annulus size. It is still unclear that normalization with BSA is the best way to predict optimal ring size or inherent annulus size for each patient. However, Gregory et al reported that, in sex-specific normalized measurement of heart and great vessel dimensions with cardiac CT angiography, normalization with BSA abolished significant differences between men and women for all measured left-sided heart and great vessel measurements, except chamber dimension, ascending aorta and left pulmonary artery sizes. ${ }^{19}$

This study has a few limitations. Basically, the database size was small and a larger study will be necessary to obtain a more accurate cut-off value. The cut-off of $18.9 \mathrm{~mm} / \mathrm{m}^{2}$ we reported here should be refined with a larger study population database. In some cases, postoperative TR grade has been increasing in mid-term of late term follow up. In addition to a large number of cases, the longer time follow up would be crucial to confirm the reliability of the TARI cut-off. Moreover, the number of larger BSA patients was relatively low in this study, and the credibility of the TARI cut-off in such patients seems to be low. We recommend the use of a 30 or $32 \mathrm{~mm}$ ring size as the upper limit of the size for the patients whose BSA is larger than $1.8 \mathrm{~m}^{2}$, as many surgeons recommend. In comparing groups $\mathrm{S}$ and L, a propensity-matched or case-matched study would have been preferable, but the relatively low number of patients in both groups precluded this statistical methodology. In particular, a large number of participants would be required to confirm the influence of each ring type in sub-analysis of group $\mathrm{S}$, and a longer-term follow up would be crucial because the advantages of rigid $3 \mathrm{D}$ rings are expected to be seen later than in this study.

Downsizing might be a potential risk to cause tricuspid stenosis after surgery, although we recommend the use of a small ring to control TR. We wanted to know the significant cut-off that results in tricuspid stenosis, but there was no postoperative TS patient in our study and that made it impossible to discuss the factor of postoperative tricuspid stenosis.

Finally, as surgeons, we occasionally feel the need for $24 \mathrm{~mm}$ rings to be implanted in patients with smaller frames, but the smallest commercially available size is $26 \mathrm{~mm}$, with just 1 company producing a $25 \mathrm{~mm}$ ring. Based on our look-up table, a $24 \mathrm{~mm}$ ring size is necessary for patients whose BSA is $<1.38 \mathrm{~m}^{2}$, to satisfy a TARI $<18.9 \mathrm{~mm} / \mathrm{m}^{2}$. We hope that our current results provide an additional incentive to ring manufacturers to add smaller sized rings to their product line.

In conclusion, we defined a novel ring size index TARI as a parameter to make it possible to discuss ring size in normalized conditions and found a threshold $18.9 \mathrm{~mm} / \mathrm{m}^{2}$ for ensuring significant TR regulation with TAP. In addition, our results suggested that ring size rather than ring properties was the most influential factor for TR control in TAP.

\section{Acknowledgement}

We wish to express our gratitude to Mr M. Tomita for his help on the review of the statistical analysis.

\section{References}

1. Antunes MJ, Barlow JB. Management of tricuspid valve regurgitation. Heart 2007; 93: 271-276.

2. Izumi C, Miyake M, Takahashi S, Matsutani H, Hashiwada S, Kuwano K, et al. Progression of isolated tricuspid regurgitation late after left-sided valve surgery: Clinical features and mechanisms. 
Circ J 2011; 75: 2902-2907.

3. Cohn LH. Tricuspid regurgitation secondary to mitral valve disease: When and how to repair. J Card Surg 1994; 9(Suppl 2): 237-241.

4. Ruel M, Rubens FD, Masters RG, Pipe AL, Bedard P, Mesana TG Late incidence and predictors of persistent or recurrent heart failure in patients with mitral prosthetic valves. J Thorac Cardiovasc Surg 2004; 128: 278-283.

5. Calafiore AM, Gallina S, Iacó AL, Contini AI, Bivona A, Gagliardi $\mathrm{M}$, et al. Mitral valve surgery for functional mitral regurgitation: Should moderate-ormore tricuspid regurgitation be treated? A propensity score analysis. Ann Thorac Surg 2009; 87: 698-703.

6. Matsuyama K, Matsumoto M, Sugita T, Nishizawa J, Tokuda Y, Matsuo T. Predictors of residual tricuspid regurgitation after mitral valve surgery. Ann Thorac Surg 2003; 75: 1826-1828.

7. Dreyfus GD, Corbi PJ, Chan KMJ, Bahrami T. Secondary tricuspid regurgitation or dilation: Which should be the criteria for surgical repair? Ann Thorac Surg 2005; 79: 127-132.

8. Nath J, Foster E, Heidenreich PA. Impact of tricuspid regurgitation on long-term survival. J Am Coll Cardiol 2004; 43: 405-409.

9. Devega NG. Selective adjustable and permanent annuloplasty: An original technique for the treatment of tricuspid insufficiency. Rev Esp Cardiol 1972; 2: 555-556.

10. Rivera R, Duran E, Ajuria M. Carpentier's flexible rings versus De Vega's annuloplasty: A prospective randomized study. J Thorac Cardiovasc Surg 1985; 89: 196-203.

11. Tang GHL, David TE, Singh SK, Maganti MD, Armstrong S, Borger MA. Tricuspid valve repair with an annuloplasty ring results in improved long-term outcomes. Circulation 2006; 114(Suppl 1):I-577I-581.

12. Jung SH, Je HG, Song JM, Choo SJ, Chung CH, Yun SC, et al. Outcomes following use of a modified Duran ring tricuspid valve reconstruction procedure for secondary tricuspid regurgitation. Circ J 2010; 74: 925-930.

13. Gatti G, Marcianò F, Antonini-Canterin F, Pinamonti B, Benussi B, Pappalardo A, et al. Tricuspid valve annuloplasty with a flexible prosthetic band. Interact Cardiovasc Thorac Surg 2007; 6: 731-736.

14. McCarthy PM, Bhudia SK, Rajeswaran J, Hoercher KJ, Lytle BW, Cosgrove DM, et al. Tricuspid valve repair: Durability and risk factors for repair. J Thorac Cardiovasc Surg 2004; 127: 674-685.

15. Fukuda S, Gillinov AM, McCarthy PM, Stewart WJ, Song JM, Kihara T, et al. Determinants of recurrent or residual functional tricuspid regurgitation after tricuspid annuloplasty. Circulation 2006; 114(Suppl 1): I-582-I-587.

16. Navia JL, Nowicki ER, Blackstone EH, Brozzi NA, Nento DE, Atik FA, et al. Surgical management of secondary valve regurgitation: Annulus, commissure, or leaflet procedure? J Thorac Cardiovasc Surg 2010; 139: $1473-1482$.
17. Ghoreishi M, Brown JM, Stauffer CE, Young CA, Byron MJ, Griffith $\mathrm{BP}$, et al. Undersized tricuspid annuloplasty rings optimally treat functional tricuspid regurgitation. Ann Thorac Surg 2011; 92: 89-96

18. Nevsky G, Jacobs JE, Lim RP, Donnino R, Babb JS, Srichai MB. Sex-specific normalized reference values of heart and great vessel dimensions in cardiac CT angiography. AJR Am J Roentgenol 2011; 196: $788-794$.

19. DuBois D, DuBois EF. A formula to estimate the approximate surface area if height and weight be known. Arch Intern Med 1916; 17: $863-871$.

20. Zoghbi WA, Enriquez-Sarano M, Foster E, Grayburn PA, Kraft CD, Levine RA, et al. Recommendations for evaluation of the severity of native valvular regurgitation with two-dimensional and Doppler echocardiography. J Am Soc Echocardiogr 2003; 16: 777-802.

21. Carpentier A. Cardiac valve surgery-the "French correction." J Thorac Cardiovasc Surg 1983; 86: 323-337.

22. Fukuda S, Gillinov AM, McCarthy PM, Matsumura Y, Thomas JD, Shiota T. Echocardiographic follow-up of tricuspid annuloplasty with a new three-dimensional ring in patients with functional tricuspid regurgitation. J Am Soc Echocardiogr 2007; 20:1236-1242.

23. Bolling SF, Deeb M, Brunsting LA, Bach DS. Early outcome of mitral valve reconstruction in patients with end-stage cardiomyopathy. J Thorac Cardiovasc Surg 1995; 109: 676-683.

24. Bax JJ, Braun J, Somer ST, Klautz R, Holman ER, Versteegh MIM, et al. Restrictive annuloplasty and coronary revascularization in ischemic mitral regurgitation results in reverse left ventricular remodeling. Circulation 2004; 110: II-103-II-108.

25. Kainuma S, Taniguchi K, Toda K, Funatsu T, Kondoh H, Nishino $\mathrm{M}$, et al. Restrictive mitral annuloplasty for functional mitral regurgitation: Acute hemodynamics and serial echocardiography. Circ J 2011; 75: 571-579.

26. Miura T, Eishi K, Yamachika S, Hashizume K, Tada S, Yamane K, et al. Isolated undersized mitral annuloplasty for functional mitral regurgitation in non-ischemic dilated cardiomyopathy: Reconsideration of the relationship between preoperative coaptation depth and persistent mitral regurgitation. Circ J 2008; 72: 1744-1750.

27. Hwang HY, Shim MS, Park EA, Ahn H. Reduction aortoplasty for the ascending aortic aneurysm with aortic valve disease: Does bicuspid valve matter? Circ J 2011; 75: 322-328.

28. Filsoufi F, Salzberg SP, Couta M, Adams DH. A three dimensional ring annuloplasty for the treatment of tricuspid regurgitation. Ann Thorac Surg 2006; 81: 2273-2278.

29. Min SY, Song JM, Kim JH, Jang MK, Kim YJ, Song H, et al. Geometric changes after tricuspid annuloplasty and predictors of residual tricuspid regurgitation: A real-time three dimensional echocardiography study. Eur Heart J 2010; 31: 2871-2880. 